\title{
A Brief Overview of Non-Celiac Gluten Sensitivity
}

\author{
Hieu Ly ${ }^{1 *} \&$ Kevin Singh ${ }^{2}$
}

1. Corresponding author. Department of Health and Rehabilitation Science, Faculty of Health Sciences, Health Promotion Research Laboratory, Western University, London, Ontario. 2. Kevin Singh, Michael G. DeGroote School of Medicine, McMaster University, Hamilton, Ontario. Email: kevin. singh@medportal.ca

The adoption of a gluten-free diet (GFD) has become an increasingly popular trend, despite the fact that most individuals do not have a diagnosis of wheat allergy (WA) or celiac disease (CD) (1). Although WA and CD are defined as immune and autoimmune conditions, respectively, certain aspects of non-celiac gluten sensitivity (NCGS) are still scrutinized by experts $(1,2)$. It is also still unclear whether NCGS is indeed an immune-mediated condition (1), especially since there is contradictory evidence on this topic (3-6). Furthermore, a lack of diagnostic tools may contribute to misdiagnoses and misunderstandings of this condition. However, many individuals with NCGS have described overall symptomatic improvements after adopting a GFD (5-7). The aim of this paper is to further explore this complex condition to better stratify patient populations.

\section{Definition, Symptoms, and Rates of NCGS}

NCGS describes individuals without WA or CD who have adverse reactions when ingesting products containing gluten, but may experience alleviation in symptoms by adopting a GFD (1). Common symptoms associated with NCGS are abdominal pain, distension, nausea, and diarrhea. Individuals may also report nonspecific extraintestinal symptoms such as headache, fatigue, rashes, and general malaise $(2,8)$. In a prospective multicenter study that included 28 centers (12,255 participants), 391 individuals were identified with suspected NCGS (3.19\%) and 340 individuals received new $C D$ diagnoses $(2.77 \%)$, which is a ratio of 1.15 to 1 (8). Additionally, researchers found that adult women are more likely to report NCGS-related symptoms than men (female to male ratio of 5.4:1), and some individuals with NCGS may also experience irritable bowel syndrome (IBS; 47\%), food intolerance (35\%), allergy (22\%), and other autoimmune disorders (14\%) (8).

\section{Diagnostic Protocol for NCGS}

Some researchers have found that NCGS is not characterized by specific antibody markers (3) or an accentuated inflammatory response to gliadin (4). In contrast, others have reported that there are detectable differences for certain biomarkers (e.g., eosinophils and immunoglobin $G)(5,6)$. The mixed findings on this topic have made it difficult for clinicians to diagnose individuals with NCGS (1). However, a two-step process for diagnosing NCGS was suggested by experts, which included guidelines that previous diagnostic procedures lacked (2). Initially, a clinician can examine patients' responsiveness to a GFD through a self-administered instrument known as the Gastrointestinal Symptom Rating Scale (2). Subsequently, patients can be introduced to a double-blind placebocontrolled gluten challenge, which includes the elimination and reintroduction of gluten in patients' diets (2). This diagnostic protocol is critical for clinicians to be able to separate NCGS from other conditions, such as IBS $(2,6,9)$, and to better understand its underlying etiologies. This is exemplified in a randomized control trial of 72 participants who were suspected of IBS, in which 31 (83.8\%) individuals on a GFD experienced fewer symptoms compared to 9 (25.7\%) individuals on the gluten-containing diet (9).

\section{The Effects of a GFD in the Treatment of NCGS}

The standard treatment for NCGS is to restrict the intake of dietary gluten (2). For example, there was a reduction in anti-gliadin antibodies and improvement in symptoms for 41 NCGS patients (93.2\%) who followed a strict compliance to a GFD for six months (5). Generally, individuals expressed an alleviation of symptoms after eliminating gluten from their diet (6), and some have reported an increase in the overall severity of their symptoms after ingesting small amounts of gluten (7). However, researchers have also reported that there were no effects of ingesting gluten among those with 
suspected NCGS, and other dietary triggers could potentially be responsible for their symptoms (3). Although there has been an expanding market for gluten-free products (1), there is a financial barrier associated with the adoption and adherence to a GFD because gluten-free products are on average $242 \%$ more expensive than regular food items (10). Therefore, it is crucial to have standardized procedures to correctly diagnose those with NCGS, before clinicians provide recommendations for individuals to adopt a GFD.

\section{Future Directions}

The increased number of cases of NCGS worldwide signals a need for standardized diagnostic procedures to determine the extent to which this condition affects the global population (1). The newly developed NCGS diagnostic protocol is a step forward to further our understanding of this complex condition. Individuals with suspected NCGS should receive a proper diagnosis from clinicians who are familiar with the diagnostic protocol prior to receiving any treatment. More evidence is needed to establish sound reasoning for advising individuals to adopt a GFD, which could potentially be a lifetime commitment. It is necessary to provide the resources (e.g., nutrition counselling from dietitians) for those who require support adopting a GFD, especially for individuals in low-income areas (10). Thus, further research on NCGS should aim to evaluate and further develop diagnostic procedures to optimize clinical decision making, and develop public health strategies to support those afflicted by NCGS worldwide.

\section{References}

1. Sapone A, Bai JC, Ciacci C, Dolinsek J, Green PH, Hadjivassiliou M, et al. Spectrum of gluten-related disorders: Consensus on new nomenclature and classification. BMC Med. 2012;10(13):1-12.

2. Catassi C, Elli L, Bonaz B, Bouma G, Carroccio A, Castillejo G, et al. Diagnosis of non-celiac gluten sensitivity (NCGS): The Salerno experts' criteria. Nutrients. 2015;7(6):4966-77.

3. Biesiekierski JR, Peters SL, Newnham ED, Rosella O, Muir JG, Gibson PR. No effects of gluten in patients with self-reported non-celiac gluten sensitivity after dietary reduction of fermentable, poorly absorbed, short-chain carbohydrates. Gastroenterol. 2013;145(2):320-28.

4. Bucci C, Zingone F, Russo I, Morra I, Tortora R, Pogna N, et al. Gliadin does not induce mucosal inflammation or basophil activation in patients with nonceliac gluten sensitivity. Clin Gastroenterol Hepatol. 2013;11(10):129499.

5. Caio G, Volta U, Tovoli F, De Giorgio R. Effect of gluten free diet on immune response to gliadin in patients with non-celiac gluten sensitivity. BMC Gastroenterol. 2014;14(26):1-7.

6. Carroccio A, Mansueto P, lacono G, Soresi M, D'Alcamo A, Cavataio F, et al. Non-celiac wheat sensitivity diagnosed by double-blind placebocontrolled challenge: Exploring a new clinical entity. Am J Gastroenterol. 2012;107(12):1898-06

7. Di Sabatino A, Volta U, Salvatore C, Biancheri P, Caio G, De Giorgio R, et al. Small amounts of gluten in subjects with suspected nonceliac gluten sensitivity: A randomized, double-blind, placebo-controlled, cross-over trial. Clin Gastroenterol Hepatol. 2015;13(9):1604-12.

8. Volta U, Bardella MT, Calabrò A, Troncone R, Corazza GR. An Italian prospective multicenter survey on patients suspected of having non-celiac gluten sensitivity. BMC Med. 2014;12(85):1-8.

9. Shahbazkhani B, Sadeghi A, Malekzadeh R, Khatavi F, Etemadi M, Kalantri E, et al. Non-celiac gluten sensitivity has narrowed the spectrum of irritable bowel syndrome: A double-blind randomized placebo-controlled trial. Nutrients. 2015;7(6):4542-54.

10. Stevens L, Mohsin R. Gluten-free and regular foods: A cost comparison. Can J Diet Pract Res. 2008:69(3):147-50.
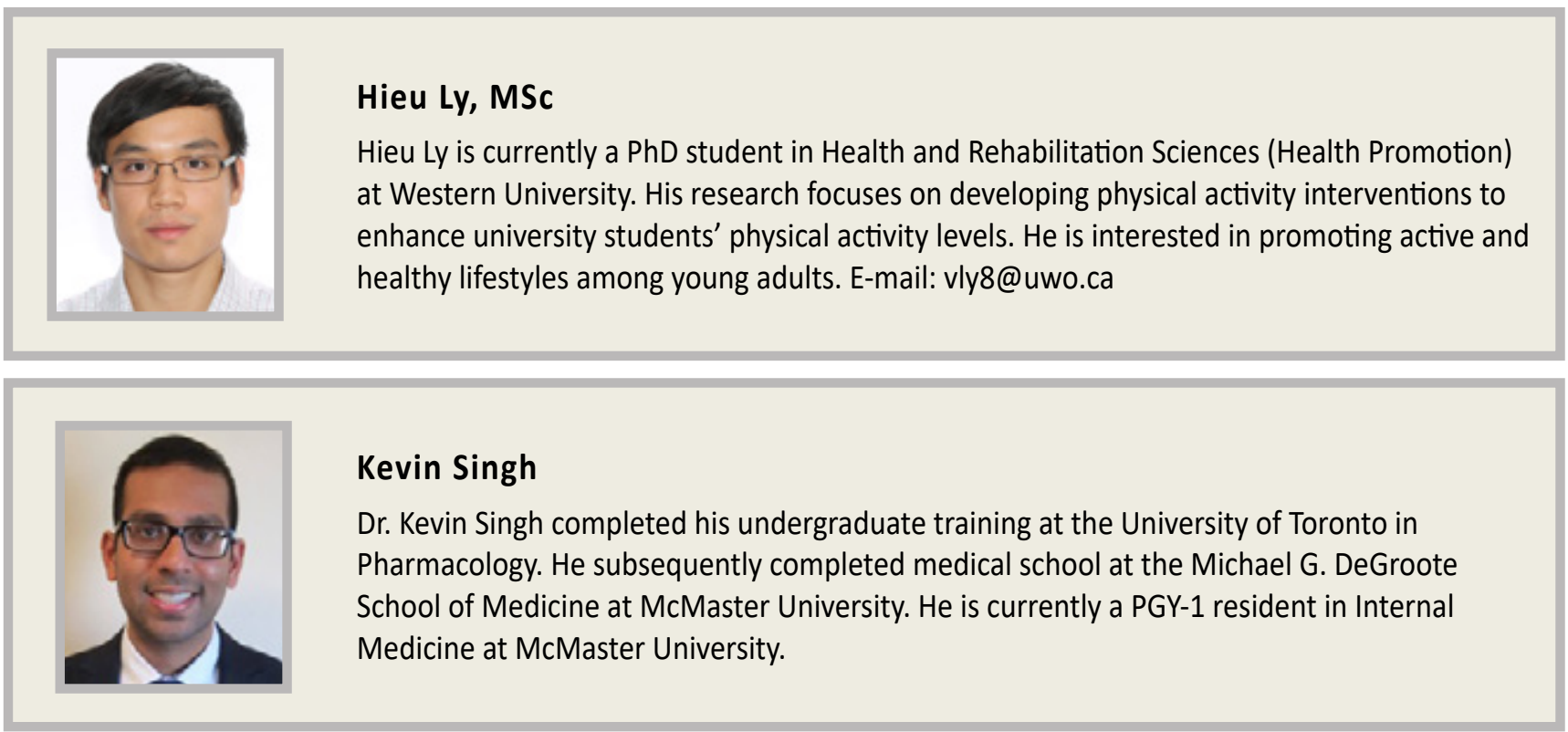